\title{
BMJ Open A smoking prevention photoageing intervention for secondary schools in Brazil delivered by medical students: protocol for a randomised trial
}

\author{
Bianca Lisa Faria, ${ }^{1}$ Christian M Brieske, ${ }^{2,3}$ Ioana Cosgarea, ${ }^{2,3}$ Albert J Omlor, ${ }^{4}$ \\ Fabian N Fries, ${ }^{4}$ Christian Olber Moreira de Faria, ${ }^{1}$ Henrique Augusto Lino, ${ }^{1}$ \\ Ana Carla Cruz Oliveira, ${ }^{1}$ Oscar Campos Lisboa, ${ }^{5}$ Joachim Klode, ${ }^{2,3}$ \\ Dirk Schadendorf, ${ }^{2,3}$ Breno Bernardes-Souza, ${ }^{5}$ Titus J Brinker ${ }^{2,3}$
}

To cite: Faria BL, Brieske CM, Cosgarea I, et al. A smoking prevention photoageing intervention for secondary schools in Brazil delivered by medical students: protocol for a randomised trial. BMJ Open 2017;7:e018589. doi:10.1136/ bmjopen-2017-018589

- Prepublication history and additional material for this paper are available online. To view these files, please visit the journal online (http://dx.doi. org/10.1136/bmjopen-2017018589).

Received 10 July 2017

Revised 31 August 2017

Accepted 22 September 2017

\section{CrossMark}

${ }^{1}$ School of Medicine, University of Itauna, Itauna, Brazil 2Department of Dermatology, Venerology and Allergology, University-Hospital Essen, University of Duisburg-Essen, Essen, Germany

${ }^{3}$ Department of Dermatology, National Center for Tumor Diseases (NCT), University Hospital Heidelberg, Heidelberg, Germany

${ }^{4}$ Saarland University Medical Center, Saarland University Faculty of Medicine, Homburg, Germany

${ }^{5}$ School of Medicine, Federal University of Ouro Preto, Ouro Preto, Brazil

Correspondence to

Titus J Brinker;

titus.brinker@gmail.com

\section{ABSTRACT}

Introduction Most smokers start smoking during their early adolescence, often with the idea that smoking is glamorous; the dramatic health consequences are too far in the future to fathom. We recently designed and tested an intervention that takes advantage of the broad availability of mobile phones as well as adolescents' interest in their appearance. A free photoageing mobile app (Smokerface) was implemented by medical students in secondary schools via a novel method called mirroring. The pupils' altered three-dimensional selfies on tablets were 'mirrored' via a projector in front of their whole grade. This is the first randomised trial to measure the effectiveness of the mirroring approach on smoking behaviour in secondary schools.

Methods and analysis The mirroring intervention, which lasts $45 \mathrm{~min}$, is implemented by Brazilian medical students in at least 35 secondary school classes with 21 participants each (at least 35 classes with 21 participants for control) in February 2018 in the city of Itauna, Brazil. External block randomisation via computer is performed on the class level with a 1:1 allocation. In addition to sociodemographic data, smoking behaviour is measured via a paper-pencil questionnaire before, 3 and 6 months postintervention plus a random carbon monoxide breathing test at baseline and end line. The primary outcome is cigarette smoking in the past week at 6 months followup. Smoking behaviour (smoking onset, quitting) and effects on the different genders are studied as secondary outcomes. Analysis is by intention to treat.

Ethics and dissemination Ethical approval is obtained from the ethics committee of the University of Itauna in Brazil. Results will be disseminated at conferences, in peerreviewed journals, throughout the Education Against Tobacco network social media channels and on our websites. Trial registration number NCT03178227.

\section{INTRODUCTION}

Most smokers start smoking during their early adolescence with the idea that smoking is glamorous; the problems related to cardiovascular disease, lung cancer and chronic

\section{Strengths and limitations of this study}

- This is the first study measuring the longitudinal effectiveness of the mirroring intervention in changing smoking behaviour.

- External randomisation via computer and a relatively high number of clusters ensure good comparability between groups.

- For the reason that intervention and control classes are located in the same schools, cluster effects cannot be excluded.

pulmonary disease are too far in the future to fathom.

After multiple failed quit attempts, many smokers end up with tobacco-related diseases doctors are unable to cure. ${ }^{1}$ Inpatient smoking cessation is effective ${ }^{2}$ and was implemented in guidelines of almost all medical specialties, ${ }^{3}$ but research has shown that about half of physicians in Brazil do not deliver it, ${ }^{4}$ especially before the onset of chronic disease. ${ }^{5}$ The problem of undertreatment of tobacco addiction is known on a global scale, ${ }^{67}$ while the behaviour of physicians in both inpatient and outpatient settings affects the subjective norm within a community, which in turn affects smoking behaviour. ${ }^{8}$

Education Against Tobacco (EAT) is a global network of medical students that aims at both: providing science-based prevention to a large number of adolescents and at the same time sensitising prospective physicians for the importance of delivering smoking cessation advice. $^{9-12}$ The network currently involves about 80 medical schools in 14 countries with 2000 medical students educating more than 40000 secondary school students in the classroom setting per year using and optimising apps and strategies. ${ }^{11-15}$ Since its foundation 


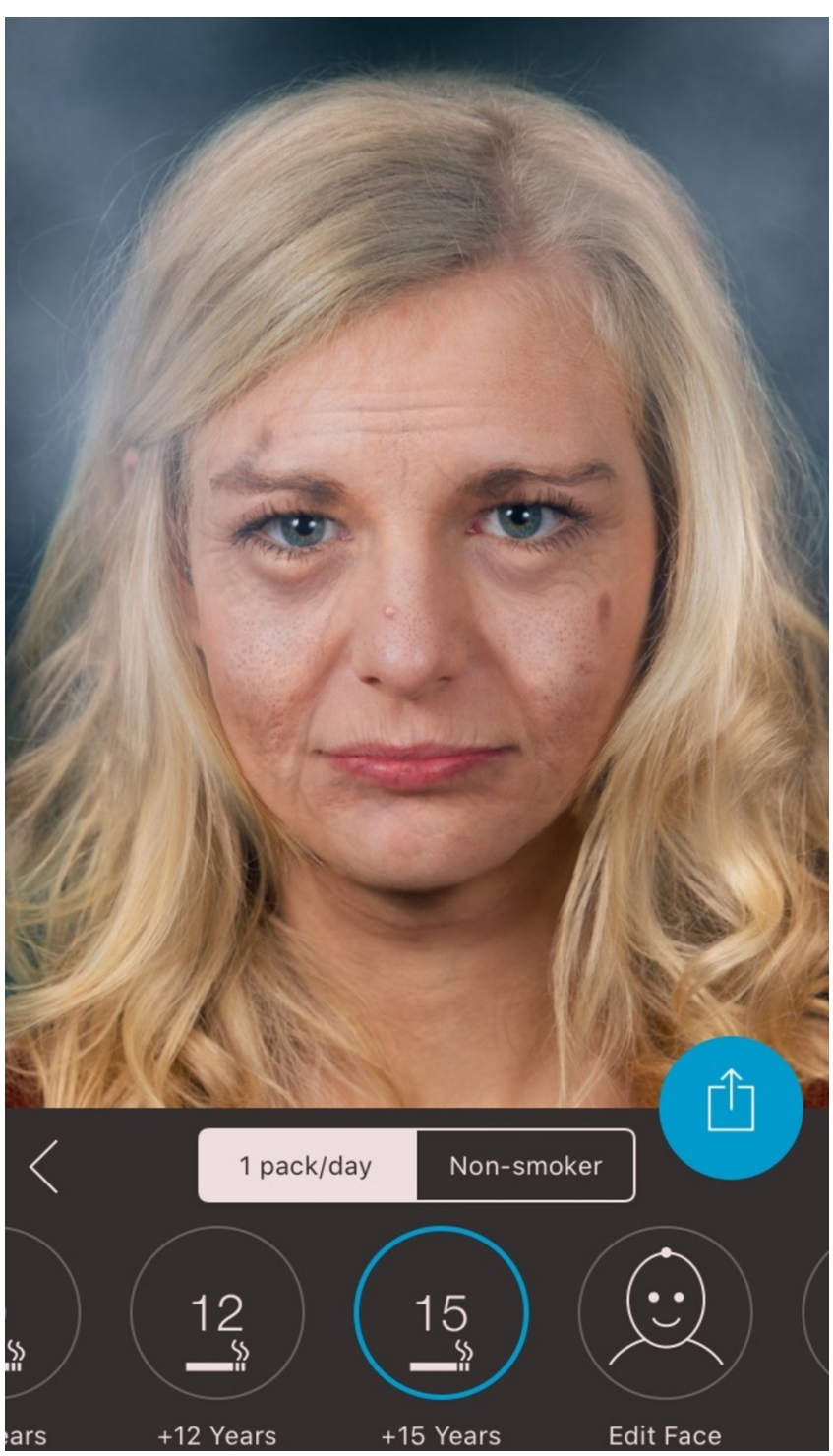

Figure 1 Effect view of normal ageing plus smoking one pack a day for 15 years.

in Germany in 2012, the network instructed sciencebased smoking cessation curricula in 14 medical schools in Germany (of the 28 medical schools in Germany that take part in EAT) currently prospectively evaluating their effectiveness in preparing medical students for inpatient smoking cessation. ${ }^{16}$ The two free science-based quit apps of EAT ('Smokerface' and 'Smokerstop') are downloaded more than 300 times per day and were translated to the most spoken languages worldwide. ${ }^{14} 15$

Baseline data from our school-based study performed in a city with regional proximity to the city of this study (Itauna) showed a baseline 30-day smoking prevalence of $14.6 \%$ in male and $13.8 \%$ in female participants in a sample size of 2038 students with an average age of 14.7 years (median=15 years; range $12-17$ years). ${ }^{11}$ National data represented in the Adolescent Cardiovascular Risk Study study were comparably lower $(5.7 \%$ of smokers among 74589 Brazilian adolescents aged from 12 to 17 years), which is explained by regional differences in smoking prevalence but also by different methods of data collection. ${ }^{17}$

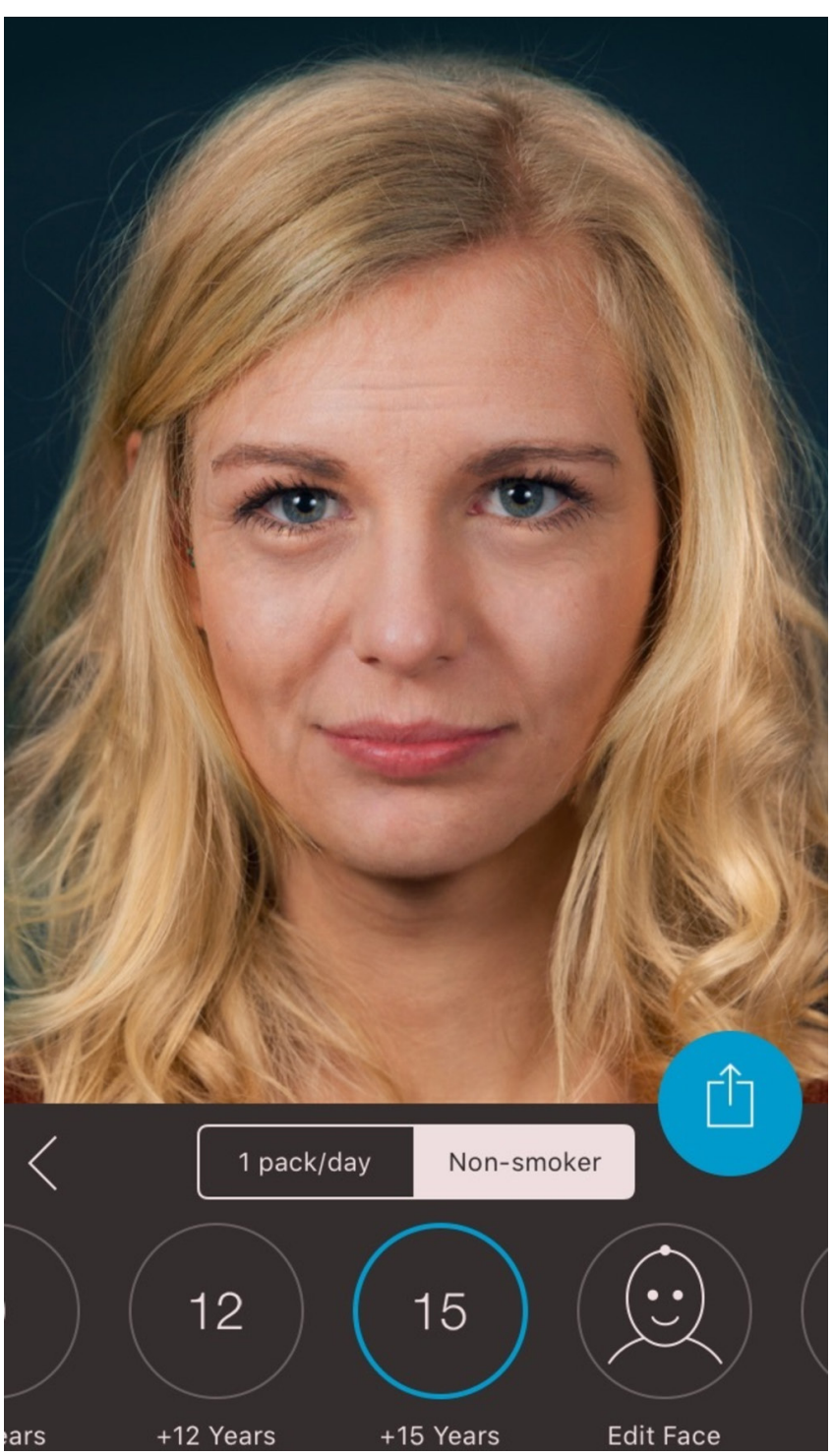

Figure 2 Effect view of normal ageing as a non-smoker for 15 years.

\section{Current knowledge on school-based tobacco prevention}

Most school-based curricula are ineffective, and data from Brazil remain scarce. ${ }^{18-20}$ The most recent trials on tobacco prevention in the school setting have focused on including school personnel or sports trainers in the intervention $^{21-23}$ and others focused on involving the families, ${ }^{24} 25$ but all of these studies concluded that the environment of the student (ie, the peer group but also parental behaviour, school policies, etc) plays a role in smoking initiation in adolescence.

Physician-based programmes relying on fear-inducing statements show no overall long-term effectiveness in reducing the smoking prevalence. ${ }^{26-29}$ A physician-based multimodal programme in Germany was evaluated in a quasi-experimental study showing significant shortterm effects in preventing smoking onset. ${ }^{30}$

In Brazil, a randomised controlled trial of different school-based interventions to reduce the use of various psychotropic substances among 1316 students from 2015 showed mixed effects for different drugs/settings 


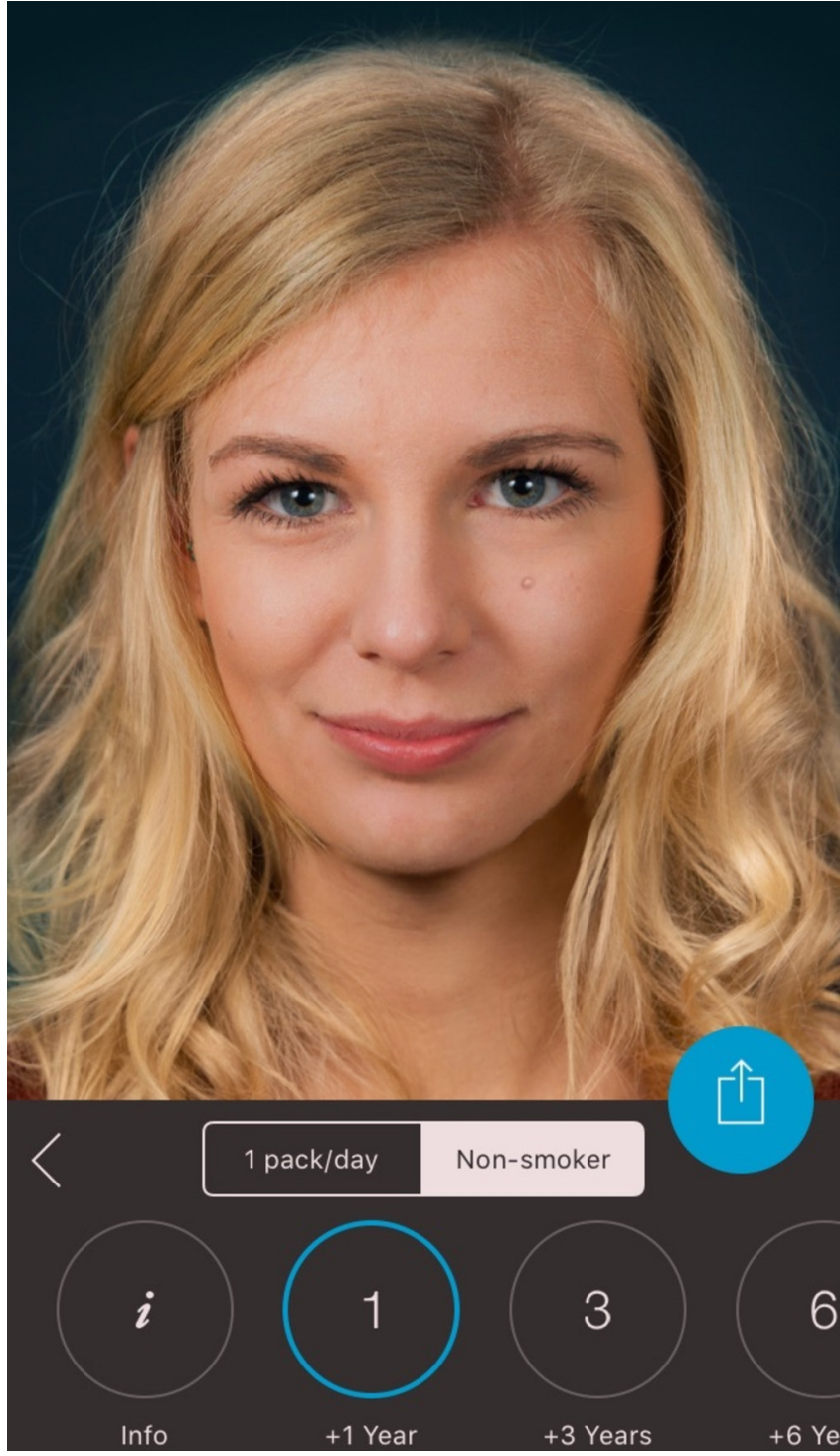

Figure 3 Normal ageing for 1 year as a non-smoker (very limited changes to the users' selfie).

with limitations of the study design that preclude interpretation. ${ }^{31}$ Another study from Brazil tried to analyse the effectiveness of an educational intervention by the Brazilian Cancer Institute (INCA) to prevent smoking among school adolescents. The researchers randomised 32 schools to either control (no intervention) or intervention arm with a total sample of 22007 th-8th graders (13-14 years old). INCA members lectured about tobacco control to teachers from schools of the intervention group, and it was expected that those teachers would discuss tobacco-related topics with their students. Questionnaires were applied before and 6 months after the interventions, and urine was obtained for biochemical validation. No change in smoking prevalence was found at end line, but knowledge on passive smoking had improved. ${ }^{32}$

\section{Introduction of the EAT app 'Smokerface'}

Photoageing desktop programmes in which an image is altered to predict future appearance were

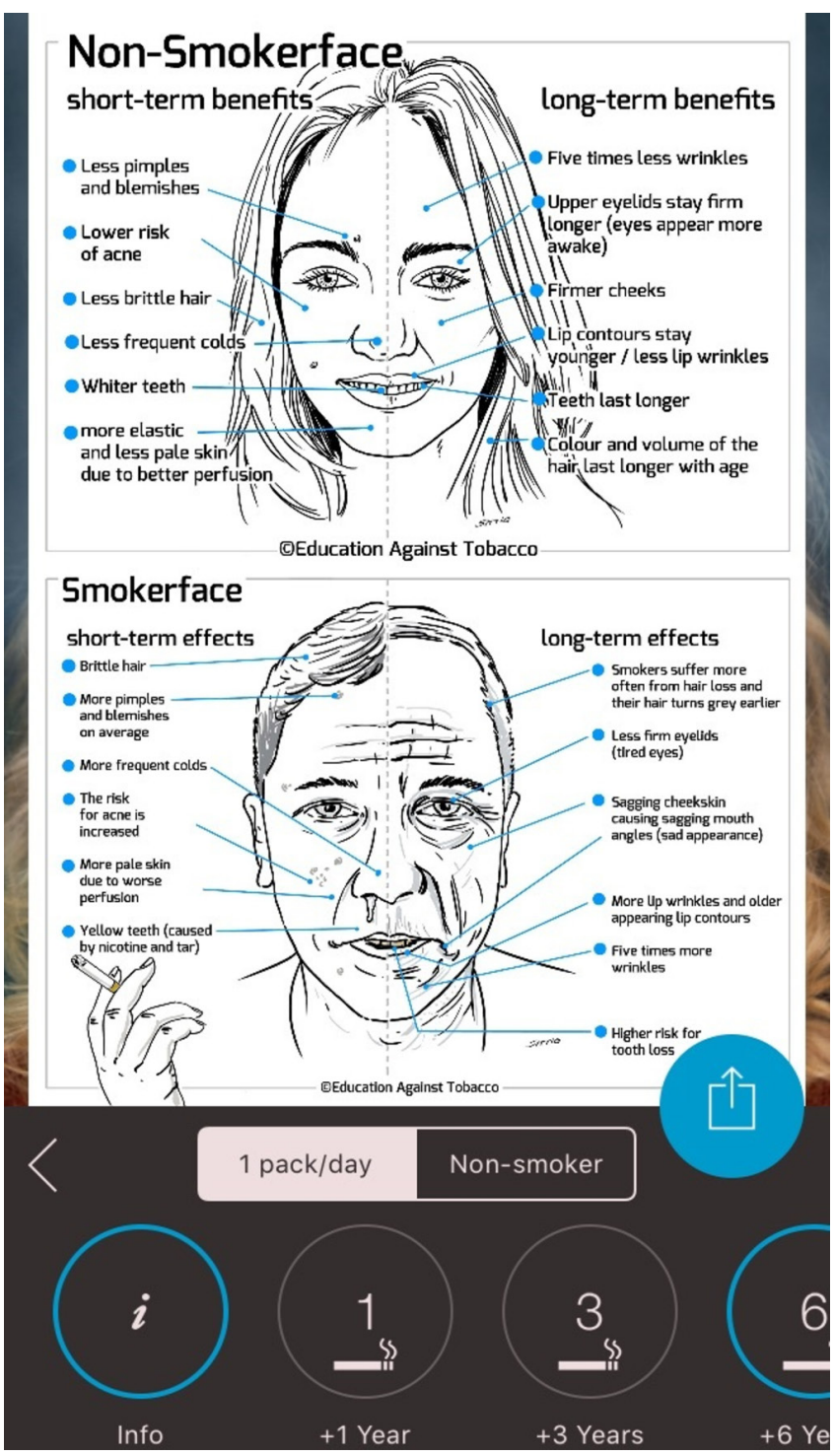

Figure 4 Explanatory graphic of the facial changes within the app.

effective in motivating 14-year-old to 18-year-old women to quit smoking and increased the rate of quit attempts in 18-year-old to 30-year-old young adults of both genders by $21 \%$ in Australia ${ }^{33} 34$ and are in line with recently published preliminary findings from France..$^{35}$ Advantage was taken of the broad availability of smartphones and adolescents' interest in appearance ${ }^{36}$ to create the free three-dimensional (3D) photoageing smartphone app 'Smokerface', ${ }^{15}$ which photoages the users' selfie into 1-year-older to 15-year-older animated versions (normal ageing vs normal ageing plus chemical photoageing due to tobacco smoke $e^{37-41}$ ) (online supplementary video and figures 1-3 explains the facial changes to the user (figure 4). It is downloaded 200 times per day and available for both iOS and Android smartphones.

\section{Previous research on photoageing in school-based tobacco} prevention

In 2014, the Smokerface app was only available as a software programme, run on notebooks that captured 
participant's faces via webcam. ${ }^{12}$ This app was implemented in the school setting by medical students as a part of the EAT intervention. While the interpretability of the results was limited by a high loss-to-follow-up of $52.3 \%$, the intervention appeared to prevent smoking, especially in women (number needed to treat $(\mathrm{NNT})=24)$ and students with a low educational background $(\mathrm{NNT}=30) .{ }^{12}$

The current 3D version of the Smokerface app is also currently implemented via a poster-campaign in German secondary schools in the context of a large multicentred trial with roughly 10000 German adolescents. ${ }^{42}$ In addition, the current app is currently integrated in a different way in a randomised trial of the EAT group in Brazil as a subpart of their school-based curriculum. $^{11}$

The mirroring intervention (the students' altered 3D selfies on tablets are 'mirrored' via a projector in front of their whole grade) under evaluation was recently conceptualised and tested in a pilot study with 125 German adolescents of both genders (average age 12.75 years) with promising results. A majority of the students perceived the intervention as fun $(77 / 125,61.6 \%)$, claimed that the intervention motivated them not to smoke $(79 / 125,63.2 \%)$ and stated that they learnt new benefits of non-smoking $(81 / 125,64.8 \%) .^{14}$

\section{Theoretical considerations on photoageing interventions in adolescence}

The self-concept of appearance, which photoageing interventions are taking advantage of, is still by far the strongest predictor of self-esteem in adolescents of both genders, which has been repeatedly demonstrated in multiple studies from different countries. ${ }^{36}{ }^{43}$ In the most recent publication by Baudson et al involving a sample of 2950 adolescents from a broad range of secondary schools, it was noted that this is especially true for students from lower educational schools and girls ${ }^{36}$ which are exactly the subgroups we measured the intervention to be most effective for. ${ }^{12}$ This may be explained by the theory of planned behaviour (TPB), according to which the subjective norm (ie, 'my friends think that smoking makes you unattractive'), the attitudes (consisting of beliefs; ie, 'smoking leads to unattractiveness') and the perceived behavioural control (ie, 'I can resist if somebody offers me a cigarette') influence both the behavioural intentions of a person and his/her behaviour. Photoageing interventions potentially affect all three of these predictors, and the mirroring intervention specifically had strong influence on the subjective norm in the previous pilot study. ${ }^{14}$

This first randomised trial was designed to answer the following main questions: is the mirroring intervention effective in changing the smoking behaviour of Brazilian adolescents of both genders? Does it sustainably alter the predictors of smoking in accordance with the TPB? ${ }^{44}$

\section{METHODS AND ANALYSIS}

\section{Trial design}

The study is designed as a randomised, controlled superiority trial with two parallel groups and a primary end point of the difference of cigarette smoking in the past week (introduced as a new gold standard in the ASSIST trial from $2008^{45}$ ) at 6 months follow-up implemented in the City of Itauna (Brazil). Randomisation is externally and centrally performed at school level with a 1:1 allocation (control to intervention) via computer. ${ }^{12}$ The intervention classes receive the Smokerface mirroring intervention, and control classes within the same schools receive no intervention. A total of at least 70 secondary school classes in Itauna, Brazil, participate in the baseline survey in February 2018. Immediately after the baseline survey, the intervention classes receive a $45 \mathrm{~min}$ lasting app-based intervention conducted by local volunteering medical students of the EAT network. Follow-up surveys are conducted 3 months and 6 months postintervention.

\section{Intervention}

To integrate app-based photoageing interventions into the school-based tobacco prevention setting, we previously developed and tested the mirroring approach in a pilot study. ${ }^{14}$ Mirroring means that the students' altered 3D selfies on smartphones or tablets are 'mirrored' via a projector in front of the whole class. The mirroring approach is implemented by volunteering medical students from the University of Itauna EAT group who receive a standardised training in advance the completion of which is monitored via a performance questionnaire by the instructor. To ensure the participation of all students within a certain class and to avoid contamination within schools, we implement the mirroring intervention via 10 Samsung Galaxy Tablets, which are already set up.

In the first $10 \mathrm{~min}$ phase, the displayed face of one student volunteer is used to show the app's altering features in the two categories (non-smoking vs smoking one pack a day for a time span of $1-15$ years) to the peer group, providing an incentive for the rest of the class to test the app. Students may interact with their own animated face via touch (sneezing, coughing, etc; see online supplementary video). In front of their peers and teachers, they could display their image as a non-smoker/ smoker (of one pack a day) 1, 3, 6, 9, 12 and 15 years in the future (see figures 1 and 2). Multiple device displays can be projected simultaneously, which are used to consolidate the altering measures with graphics (eg, to explain the chemical photoageing caused by smoking). We implement mirroring with Galaxy Tab A (Samsung) via Apple's proprietary AirPlay interface using the Android app 'Mirroring360' (Splashtop).

After the demonstration of the functionality in the first phase with one student volunteer, in the second $15 \mathrm{~min}$ phase, all present students are encouraged to try the app on one of the tablet computers, which are connected to the beamer in front. The number of provided tablet computers was calculated so the phase would take up 
to $12 \mathrm{~min}$ at the most, factoring in a utilisation time of about $4 \mathrm{~min}$ per student. By this calculation, $25 \mathrm{~min}$ of the mirroring intervention and 10 provided tablets were sufficient to have every student within a class of 40 pupils successfully mirrored at least once.

In the following $15 \mathrm{~min}$, the remaining functions of the app are discussed with the students. Facial changes, quitting via the free Smokerstop app and impaired growth, strength and sagginess of women's breasts are discussed in an interactive setting. These topics are strictly in line with the explanatory graphics within the app.

In the last $5 \mathrm{~min}$, the perception of the intervention by the students is measured directly after the intervention via three items in reference to our previous cross-sectional survey ${ }^{14}$ in an anonymous questionnaire on 5-point Likert scales: (1) 'The animation of my 3D selfie motivates me to remain a non-smoker,' (2) 'I learned new benefits of non-smoking' and (3) 'The intervention was fun.'

\section{PARTICIPANTS}

\section{Eligibility criteria at baseline}

Students from Itauna in south Brazil attending grades 6-12 in regular secondary schools are eligible.

\section{Contaminated classes}

The final intention-to-treat analysis will contain all classes that participated at baseline. We will assess app use in both groups at 6 months follow-up to assess contamination of control classes and which will be the basis for a secondary (sensitivity) analysis with the met hods described in the Analysis section of this protocol.

\section{Procedure}

Data are collected via a paper pencil questionnaire. In addition, smoking status is validated by a random carbon monoxide (CO) breathing test at baseline and end line. In addition to sociodemographic data (age, gender and school type), the questionnaire captures the smoking status of the school students concerning cigarettes and multiple other tobacco products (such as e-cigarettes and waterpipes) and was used as well as tested in previous studies in Germany and Brazil. ${ }^{11} 1242$ In addition, the questionnaire captures the change of behavioural predictors in accordance with the TPB that we used in our previous cross-sectional study. ${ }^{14}$ As not all predictors can be monitored due to the fact that this would add length to the questionnaire, which leads to frustration of some of the students in our pretest and complaints by teachers that found the time needed for data collection too long, we only included the two most important ones in the context of our study which are (1) change in subjective norm (due to peer-group effects) and (2) intention to change behaviour. Since Portuguese equivalents were not available for all of the items used, we used the Conceptual Method for translation described by the WHO/United Nations Economic and Social Commission for Asia and the Pacific Project on Health and Disability Statistics. ${ }^{46}$
Newly translated and/or modified items were extensively pretested and subjected to statistical analyses. ${ }^{42}$

\section{Biochemical validation}

Using a portable $\mathrm{CO}$ analyser (Smokerlyzer piCO+, Bedfont Scientific), $\mathrm{CO}$ testing will be randomly performed at baseline and end line in at least $10 \%$ of students. ${ }^{47} \mathrm{CO}$ measurements will be conducted by medical students with recordings made in the afternoon. The cut-off point is defined as 6 parts per million or less for non-smoking and more than 6 parts per million for smoking. ${ }^{48}$

\section{Data collection}

Each data collector involved receives training for data collection and is required to use an adapted standardised protocol for data collection, an optimised version of the one used in the Smokerface randomised trial. ${ }^{42} \mathrm{~A}$ total of 70 classes are included into the study. Written informed consent was obtained from all participants and their parents.

\section{Cluster randomisation}

In accordance with the guidelines for good epidemiological practice, classes within schools are externally and centrally randomly assigned to the control or intervention group via block randomisation in a ratio of 1:1 (control to intervention) via computer by a statistician at the University of Duisburg-Essen, Germany.

\section{Outcomes}

The primary end point is defined as the difference in smoking prevalence in the past week at the 6-month follow-up between the two groups. The difference of the 7-day/30-day and daily smoking prevalence between the two groups at 3 months, the 30 -day smoking and daily prevalence at 6 months, the number of new smokers, the number of quitters and the change in the number of never-smokers after 3 and 6 months will be compared between the two groups as secondary outcomes. For all end points, the NNT will be calculated. A smoker is defined as a pupil who claims to have smoked cigarettes at least 1 day in the 7 days preceding the survey. Those pupils who claim not to have smoked cigarettes in the past 7 days are defined as non-smokers. All participants who claim to have smoked more than once per week in the past are defined as ex-smokers.

\section{Statistical considerations}

\section{Sample size calculation}

We calculated sample sizes of 735 for both groups, which were obtained by sampling 35 clusters with 21 subjects each in the intervention group and in the control group to achieve $80 \%$ power to detect a prevalence difference between the groups of $4 \%$ taking into account an intraclass correlation of 0.033 calculated from our previous study. ${ }^{10}$ The 7-day smoking prevalence was assumed to be $8 \%$ under the null hypothesis and $12 \%$ under the alternative hypothesis. The test statistic used is the two-sided score test (Farrington and Manning). The significance 
level of the test was 0.05. Normal class size in Brazil is 35 pupils, and thus, a loss-to-follow-up effect of $40 \%$ was taken into account.

\section{Data entry}

Data entry is performed manually at the University of Itauna in Brazil into a Microsoft Excel sheet by locally trained personnel.

\section{Analysis}

To examine baseline differences in pupils' characteristics in our experimental design, we will use $\chi^{2}$ tests for the categorical variables and Student's t-tests for continuous variables. To test for differences in baseline and follow-up smoking prevalence between groups, we will use a cluster-adjusted Mantel-Haenszel $\chi^{2}$ test $^{49}$ at a significance level of $5 \%$ (two sided). For the main analysis, hierarchical linear models (HLMs) will be applied. HLM can handle the nested structure of the data and will be used to test for between-group differences in within-group changes in smoking behaviour over time. HLM will also be used to investigate the influence of further covariates and time-dependent behaviour in secondary analyses. Statistical analyses will be performed using the version 24 of SPSS Statistics (IBM, New York, New York, USA).

The effect that missing data might have on results will be assessed via sensitivity analysis. Drop-outs (essentially participants who withdraw consent for continued follow-up or who are missing in the classroom during the survey) will be included in the analysis by modern imputation methods for missing data and multiple imputation will be used to estimate treatment effect. ${ }^{50}$

\section{DISCUSSION}

\section{Principal considerations}

While cross-sectional data on the mirroring intervention exist, ${ }^{14}$ this is the first study measuring its longitudinal effectiveness in changing smoking behaviour. Based on data from a trial that implemented photoageing (without mirroring) as a subpart of a smoking prevention programme (and has thus to be interpreted with caution), photoageing interventions work specifically well in female adolescents. ${ }^{12}$ Apart from smoking prevention, photoageing has been evaluated in other behavioural change settings such as smoking cessation $^{3351-53}$ but also tanning ${ }^{54-58}$ and adiposity prevention ${ }^{5960}$ and showed promising results in all of these behavioural change settings.

The mobile phone app we investigate in this trial is easy to implement and was well received in our prior pilot study. ${ }^{14}$ It is evaluated in a way that does not go beyond what the app itself provides from an educational standpoint. This is important, as this makes the data interpretable for the mirroring intervention itself (and not other aspects one might add in other school-based tobacco prevention programme).

\section{Generalisability}

As this study is conducted only in Brazil, our results might not be generalisable to other cultural settings. However, WHO is concerned about the tobacco industry targeting young women in their advertisements, especially in developing and emerging countries in which cosmetic products, as well as tobacco use, are on the rise. ${ }^{61}$ These developments increase the global relevance of our research.

\section{Limitations}

As the control and intervention classes are in the same schools, cluster effects cannot be excluded entirely and methods of containing contamination limit the intensity of our intervention (ie, giving the app name to the students). Smoking status is biochemically validated via a random CO test in $10 \%$ of the students. Other studies used cotinine testing in urine or saliva, which was proposed as the gold standard by Connor Gorber et al in 2009. ${ }^{62}$ However, this method is not able to distinguish between e-cigarettes and regular cigarettes. Also, it is more costly and more invasive than $\mathrm{CO}$ testing and thus might be outdated in schoolbased settings. Combining $\mathrm{CO}$ and cotinine saliva would be the most sensitive (but also most expensive and complicated) method for a definitive trial. ${ }^{63}$ The limitations of $\mathrm{CO}$ testing may be found elsewhere. ${ }^{48}$ Lastly, our process evaluation on the complete delivery of the training relies on the self-reported completion of the standardised training by the medical students as well as on the limited data based on the TPB that we receive from the adolescents. An optimal process evaluation would also cover teachers and would cover all aspects of the TPB which is, however, not possible in the public school setting in Brazil.

In summary, we present the first longitudinal study measuring if the mirroring intervention prevents smoking. With regards to the available literature, we anticipate a stronger effect for female students than for male students. At the same time, the intervention engages prospective physicians into tobacco control activities.

\section{Ethics and dissemination}

Written informed consent is obtained by the data collectors from both the participants themselves and their parents. All participant information will be stored in locked file cabinets in areas with limited access. Participants' study information will not be released outside of the study without the written permission of the participant. Results will be disseminated at conferences, in peer-reviewed journals, on our websites and throughout the multinational EAT network.

Acknowledgements The authors thank all participating medical students, teachers, parents, students and schools in Itauna.

Contributors TJB initiated the study, invented, designed and organised the intervention, wrote the manuscript, drafted the design of the study and performs the statistical analyses. BB-S participated in conception of the study. JK, FNF, IC, AJO, CMB and DS contributed to the design of the study and analyses of data and proof-read the manuscript. BBS and OCL contributed to the design and logistics of the study, assisted with the translation of classroom materials and reviewed the final version of the manuscript. BLF, OMF, HAL and ACCO conduct data entry and coordinate/conduct the intervention in Brazil, supported the translation 
of the classroom materials and proofread the manuscript. All authors declare responsibility for the integrity of data and findings presented and have full access to the final trial dataset.

Funding The tablets were provided by Education Against Tobacco (Essen, Germany) that had previously received them for a different trial sponsored by the German Lung Foundation (Langenhagen, Germany) where they were not needed anymore. All questionnaires and logistics are locally sponsored by the University of Itauna in Brazil.

Competing interests None declared.

Patient consent Detail has been removed from this case description/these case descriptions to ensure anonymity. The editors and reviewers have seen the detailed information available and are satisfied that the information backs up the case the authors are making.

Ethics approval Ethics committee at the University of Itauna, Itauna, Brazil.

Provenance and peer review Not commissioned; externally peer reviewed.

Open Access This is an Open Access article distributed in accordance with the Creative Commons Attribution Non Commercial (CC BY-NC 4.0) license, which permits others to distribute, remix, adapt, build upon this work non-commercially, and license their derivative works on different terms, provided the original work is properly cited and the use is non-commercial. See: http://creativecommons.org/ licenses/by-nc/4.0/

(C) Article author(s) (or their employer(s) unless otherwise stated in the text of the article) 2017. All rights reserved. No commercial use is permitted unless otherwise expressly granted.

\section{REFERENCES}

1. Goodchild M, Nargis N, Tursan d'Espaignet E. Global economic cost of smoking-attributable diseases. Tob Control 2017:tobaccocontrol-2016-053305.

2. Bell R, Glinianaia SV, Waal ZV, et al. Evaluation of a complex healthcare intervention to increase smoking cessation in pregnant women: interrupted time series analysis with economic evaluation. Tob Control 2017:tobaccocontrol-2016-053476.

3. Batra A, Petersen KU, Hoch E, et al. S3 guideline "screening, diagnostics, and treatment of harmful and addictive tobacco use". Sucht 2016;62:139-52.

4. Abdullah AS, Stillman FA, Yang L, et al. Tobacco use and smoking cessation practices among physicians in developing countries: a literature review (1987-2010). Int J Environ Res Public Health 2013;11:429-55.

5. Balmford J, Leifert JA, Jaehne A. 'Tobacco dependence treatment makes no sense because': rebuttal of commonly-heard arguments against providing tobacco dependence treatment in the hospital setting. BMC Public Health 2014;14.

6. Bernstein SL, Yu S, Post LA, et al. Undertreatment of tobacco use relative to other chronic conditions. Am J Public Health 2013;103:e59-e65.

7. Richter KP, Ellerbeck EF. It's time to change the default for tobacco treatment. Addiction 2015;110:381-6.

8. Chow CK, Corsi DJ, Gilmore AB, et al. Tobacco control environment: cross-sectional survey of policy implementation, social unacceptability, knowledge of tobacco health harms and relationship to quit ratio in 17 low-income, middle-income and high-income countries. BMJ Open 2017;7.

9. Brinker TJ, Stamm-Balderjahn S, Seeger W, et al. Education Against Tobacco (EAT): a quasi-experimental prospective evaluation of a multinational medical-student-delivered smoking prevention programme for secondary schools in Germany. BMJ Open 2015;5.

10. Brinker TJ, Stamm-Balderjahn S, Seeger W, et al. Education Against Tobacco (EAT): a quasi-experimental prospective evaluation of a programme for preventing smoking in secondary schools delivered by medical students: a study protocol. BMJ Open 2014;4.

11. Xavier LE, Bernardes-Souza B, Lisboa OC, et al. A medical studentdelivered smoking prevention program, education against tobacco, for secondary schools in Brazil: study protocol for a randomized trial. JMIR Res Protoc 2017;6.

12. Brinker TJ, Owczarek AD, Seeger W, et al. A medical studentdelivered smoking prevention program, education against tobacco, for secondary schools in Germany: randomized controlled trial. J Med Internet Res 2017;19.

13. Education against tobacco international. http://educationtobacco. org/ (accessed 13 Mar 2016).
14. Brinker TJ, Seeger W, Buslaff F. Photoaging mobile apps in schoolbased tobacco prevention: the mirroring approach. J Med Internet Res 2016;18.

15. Brinker TJ, Seeger W. Photoaging mobile apps: a novel opportunity for smoking cessation? J Med Internet Res 2015;17.

16. Strobel L, Schneider NK, Krampe H, et al. German medical students lack knowledge of how to treat smoking and problem drinking. Addiction 2012;107:1878-82.

17. Figueiredo VC, Szklo AS, Costa LC, et al. ERICA: smoking prevalence in Brazilian adolescents. Rev Saude Publica 2016;50.

18. Thomas RE, McLellan J, Perera R. Effectiveness of school-based smoking prevention curricula: systematic review and meta-analysis. BMJ Open 2015;5:e006976.

19. Peirson L, Ali MU, Kenny M, et al. Interventions for prevention and treatment of tobacco smoking in school-aged children and adolescents: a systematic review and meta-analysis. Prev Med 2016;85:20-31.

20. Hefler M, Liberato SC, Thomas DP. Incentives for preventing smoking in children and adolescents. Cochrane Database Syst Rev 2017;6:CD008645.

21. Bast LS, Due P, Ersbøll AK, et al. Association of school characteristics and implementation in the X:IT study-a school-randomized smoking prevention program. J Sch Health 2017;87:329-37.

22. Midford R, Cahill H, Lester L, et al. Smoking prevention for students: findings from a three-year program of integrated harm minimization school drug education. Subst Use Misuse 2016;51:395-407.

23. Garnham-Lee K, Trigwell J, McGee CE, et al. Impact and acceptability of the coach and teacher Ttraining within a schoolbased sport-for-health smoking prevention intervention: smoke free sports. J Child Adolesc Subst Abuse 2016;25:606-12.

24. Brown N, Luckett T, Davidson PM, et al. Family-focussed interventions to reduce harm from smoking in primary schoolaged children: a systematic review of evaluative studies. Prev Med 2017;101:117-25.

25. Chan SS, Cheung YT, Fong DY, et al. Family-based smoking cessation intervention for smoking fathers and nonsmoking mothers with a child: a randomized controlled trial. J Pediatr 2017;182:260-6.

26. Kreuter M, Bauer CM, Ehmann M, et al. Efficacy and sustainability of a smoking prevention program for pupils-ohnekippe. Dtsch Med Wochenschr 2014;139.

27. Thrul J, Bühler A, Herth FJF. Prevention of teenage smoking through negative information giving, a cluster randomized controlled trial. Drugs 2014;21:35-42.

28. Sack P-M, Hampel J, Bröning S, et al. Was limitiert schulische tabakprävention? Prävention und Gesundheitsförderung 2013;8:246-51.

29. Kok G, Bartholomew LK, Parcel GS, et al. Finding theory- and evidence-based alternatives to fear appeals: Intervention Mapping. Int J Psychol 2014;49:98-107.

30. Stamm-Balderjahn S, Groneberg DA, Kusma B, et al. Smoking prevention in school students. Dtsch Ärzteb Int 2012;109.

31. do Nascimento MO, De Micheli D. Evaluation of different schoolbased preventive interventions for reducing the use of psychotropic substances among students: a randomized study. Cien Saude Colet 2015;20:2499-510.

32. Malcon MC, Menezes A, Assunção MCF, et al. Effectiveness of aneducational intervention on smoking among school adolescents. Rev. bras. epidemiol 2011;14:63-72.

33. Burford $\mathrm{O}$, Jiwa $\mathrm{M}$, Carter $\mathrm{O}$, et al. Internet-based photoaging within Australian pharmacies to promote smoking cessation: randomized controlled trial. J Med Internet Res 2013;15:e64.

34. Weiss C, Hanebuth D, Coda P, et al. Aging images as a motivational trigger for smoking cessation in young women. Int $J$ Environ Res Public Health 2010;7:3499-512.

35. Burford O, Kindarji S, Parsons R, et al. Using visual demonstrations in young adults to promote smoking cessation: preliminary findings from a French pilot study. Res Social Adm Pharm 2017.

36. Baudson TG, Weber KE, Freund PA. More than only skin deep: appearance self-concept predicts most of secondary school students' self-esteem. Front Psychol 2016;7.

37. Chien AL, Qi J, Cheng N, et al. Perioral wrinkles are associated with female gender, aging, and smoking: Development of a genderspecific photonumeric scale. J Am Acad Dermatol 2016;74:924-30.

38. Jamal BT. The effect of smoking on facial aging among females in Saudi Arabia. J Clin Exp Dermatol Res 2017;4:1-4.

39. Schäfer T, Nienhaus A, Vieluf D, et al. Epidemiology of acne in the general population: the risk of smoking. Br J Dermatol 2001;145:100-4.

40. Okada HC, Alleyne B, Varghai K, et al. Facial changes caused by smoking: a comparison between smoking and nonsmoking identical twins. Plast Reconstr Surg 2013;132:1085-92. 
41. Krutmann J, Bouloc A, Sore G, et al. The skin aging exposome. J Dermatol Sci 2017;85:152-61.

42. Brinker TJ, Holzapfel J, Baudson TG, et al. Photoaging smartphone app promoting poster campaign to reduce smoking prevalence in secondary schools: the smokerface randomized trial: design and baseline characteristics. BMJ Open 2016;6:e014288.

43. Wichstrøm L, von Soest T. Reciprocal relations between body satisfaction and self-esteem: a large 13-year prospective study of adolescents. J Adolesc 2016;47:16-27.

44. Ajzen I. Theory of planned behavior. Handb Theor Soc Psychol 2011;1:438

45. Campbell R, Starkey F, Holliday J, et al. An informal school-based peer-led intervention for smoking prevention in adolescence (ASSIST): a cluster randomised trial. Lancet 2008;371:1595-602.

46. Robine J, Jagger C. Translation \& linguistic evaluation protocol \& supporting material. WHO/UNESCAP Projecton Health and Disability Statistics, 2003.

47. Adams J, Parkinson L, Sanson-Fisher RW, et al. Enhancing selfreport of adolescent smoking: the effects of bogus pipeline and anonymity. Addict Behav 2008;33:1291-6.

48. Cropsey KL, Trent LR, Clark CB, et al. How low should you go? Determining the optimal cutoff for exhaled carbon monoxide to confirm smoking abstinence when using cotinine as reference. Nicotine Tob Res 2014;16:1348-55.

49. Donald A, Donner A. Adjustments to the mantel-haenszel chisquare statistic and odds ratio variance estimator when the data are clustered. Stat Med 1987;6:491-9.

50. Sterne JA, White IR, Carlin JB, et al. Multiple imputation for missing data in epidemiological and clinical research: potential and pitfalls. BMJ 2009;338:b2393.

51. Burford O, Smith M, Jiwa M, et al. Photoageingintervention (PAINT): a proposal for a randomised controlled trial inAustralian primary care. Austalasian Medical Journal 2009;1:8-12.

52. Flett K, Grogan S, Clark-Carter D, et al. Male smokers' experiences of an appearance-focused facial-ageing intervention. $J$ Health Psychol 2017;22:422-33.
53. Brinker TJ, Enk A, Gatzka M, et al. A Dermatologist's Ammunition in the War Against Smoking: A Photoaging App. J Med Internet Res 2017;19:e326.

54. Stapleton JL, Hillhouse J, Levonyan-Radloff K, et al. Review of interventions to reduce ultraviolet tanning: need for treatments targeting excessive tanning, an emerging addictive behavior. Psychol Addict Behav 2017.

55. Heckman CJ, Handorf EA, Darlow SD, et al. An online skin cancer risk-reduction intervention for young adults: mechanisms of effects. Health Psychol 2017:36:215-25.

56. Görig T, Diehl K, Greinert R, et al. Prevalence of sun-protective behaviour and intentional sun tanning in German adolescents and adults: results of a nationwide telephone survey. J Eur Acad Dermatol Venereol 2017.

57. Brinker TJ, Schadendorf D, Klode J, et al. Photoaging mobile apps as a novel opportunity for melanoma prevention: pilot study. JMIR Mhealth Uhealth 2017;5:e101.

58. Brinker TJ, Brieske CM, Schaefer CM, et al. Photoaging Mobile Apps in School-Based Melanoma Prevention: Pilot Study. J Med Internet Res 2017;19:e319.

59. Jiwa M, Burford O, Parsons R. Preliminary findings of how visual demonstrations of changes to physical appearance may enhance weight loss attempts. Eur J Public Health 2015;25:283-5.

60. Ossolinski G, Jiwa M, McManus A, et al. Do images of a personalised future body shape help with weight loss? A randomised controlled study. Trials 2017;18:180.

61. World Health Organization WHO. WHO report on theglobal tobacco epidemic, 2015: raising taxes on tobacco, 2015.

62. Connor Gorber S, Schofield-Hurwitz S, Hardt J, et al. The accuracy of self-reported smoking: a systematic review of the relationship between self-reported and cotinine-assessed smoking status. Nicotine Tob Res 2009;11:12-24.

63. Naughton F, Jamison J, Boase S, et al. Randomized controlled trial to assess the short-term effectiveness of tailored web- and text-based facilitation of smoking cessation in primary care (iQuit in practice). Addiction 2014;109:1184-93. 
Correction: A smoking prevention photoageing intervention for secondary schools in Brazil delivered by medical students: protocol for a randomised trial

Faria BL, Brieske CM, Cosgarea I, et al. A smoking prevention photoageing intervention for secondary schools in Brazil delivered by medical students: protocol for a randomised trial. BMJ Open 2017;7:e018589. doi: 10.1136/bmjopen-2017-018589.

The author name 'Olber Moreira de Faria' should be 'Christian Olber Moreira de Faria'.

Open Access This is an Open Access article distributed in accordance with the Creative Commons Attribution Non Commercial (CC BY-NC 4.0) license, which permits others to distribute, remix, adapt, build upon this work non-commercially, and license their derivative works on different terms, provided the original work is properly cited and the use is non-commercial. See: http://creativecommons.org/licenses/by-nc/4.0/

C Article author(s) (or their employer(s) unless otherwise stated in the text of the article) 2018. All rights reserved. No commercial use is permitted unless otherwise expressly granted.

BMJ Open 2018;8:e018589corr1. doi:10.1136/bmjopen-2017-018589corr1

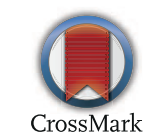

